[0212-7199 (2006) 23: 11; pp 546-551] ANALES DE MEDICINA INTERNA Copyright $($ C 2006 ARAN EDICIONES, S.L.

An. Med. InTERna (Madrid) Vol. 23, N. ${ }^{\circ} 11$, pp. 546-551, 2006

\section{Diagnóstico diferencial de las gammapatías monoclonales}

\author{
M. J. MOLINA GARRIDO, C. GUILLÉN PONCE, M. GUIRADO-RISUEÑO, \\ C. MARTÍNEZ Y SEVILA, A. CARRATO MENA
}

Servicio de Oncología Médica. Hospital General Universitario de Elche. Alicante.

MONOCLONAL GAMMOPATHIES DIFFERENTIAL DIAGNOSIS

\begin{abstract}
RESUMEN
Las aparición de un pico monoclonal en sangre o en orina, es un hallazgo que aparece con bastante frecuencia en la práctica clínica habitual. Son múltiples las patologías que pueden ser responsables de tal dato, y conseguir llegar al diagnóstico definitivo determinará que el tratamiento sea el adecuado y que se modifique el pronóstico del paciente. Asimismo, el descartar una etiología maligna de la gammapatía monoclonal, permitirá evitar pruebas cruentas innecesarias a este grupo de pacientes. En el siguiente manuscrito, se ofrece una descripción de las principales patologías asociadas a picos monoclonales, así como datos que orientarán al diagnóstico definitivo.
\end{abstract}

PALABRAS CLAVE: Pico monoclonal. Electroforesis. Gammapatía monoclonal de significado incierto.

\begin{abstract}
The presence of a monoclonal pike in blood is very habitual in our daily medical activity. There are a lot of causes of that. If we know which kind of pathology had originated the monoclonal pike, we could be able to bring a suitable treatment and to change the prognosis of the patient. If we are able to exclude a malignant disease, the patient will not suffer unnecessary aggressive tests. In this report, we describe the main diseases which are associated to monoclonal pike and the clues to get a definitive diagnosis.
\end{abstract}

KEY WORDS: Monoclonal pike. Electrophoresis. Monoclonal gammopathy of unknown origin.

Molina Garrido MJ, Guillén Ponce C, Guirado-Risueño M, Martínez y Sevila C, Carrato Mena A. Diagnóstico diferencial de las gammapatías monoclonales. An Med Interna (Madrid) 2006; 23: 546-551.

\section{INTRODUCCIÓN}

Las gammapatías son aquel grupo de enfermedades caracterizado por la proliferación de las células que sintetizan las inmunoglobulinas; casi siempre, esto conduce a la aparición de un componente monoclonal; en estos caos se habla de gammapatía monoclonal (GM). No siempre se trata de procesos malignos, sino que en ocasiones pueden ser benignos. En la tabla I, se indica la clasificación de las gammapatías y de los distintos procesos asociados a las mismas.

\section{¿CÓMO RECONOCER UNA GAMMAPATÍA MONOCLONAL?}

Puede existir un pico monoclonal de inmunoglobulinas. Aunque la concentración total de proteínas séricas, los niveles de globulinas beta y gamma, y los valores cuantitativos de Ig sean normales. Los análisis de sangre o de orina constituyen un método de screening rápido y sensible para detectar la presencia de una inmunoglobulina monoclonal y el tipo. La electroforesis en gel de agarosa (en sangre y en orina) es el método preferido para su detección. La inmunoglobulina monoclonal suele identificarse por un pico alto y delgado en la región beta o en la gamma, y más rara vez, en la región de la globulina alfa 2. Un aumento policlonal de las inmunoglobulinas, por el contrario, produce una banda ancha en la región gamma. Una vez detectado el pico monoclonal mediante la electroforesis, debe realizarse la inmunofijación o inmunoelectroforesis (en sangre y en orina), con la que se identificará el tipo de inmunoglobulina predominante (tanto el tipo de cadena pesada como el tipo de cadena ligera). Esta técnica también debe realizarse cuando la electroforesis no haya sido diagnóstica, y persista la sospecha de la existencia de una gammapatía monoclonal.

Existe numerosas causas por las que puede pasar desapercibido un pico monoclonal: si éste es pequeño, ya que puede

Trabajo aceptado: 5 de junio de 2006

Correspondencia: María José Molina Garrido. Servicio de Oncología Médica. Hospital General Universitario. Camino de la Almazara, 11. 03230 Elche (Alicante).e-mail: mjmolinagarrido@ hotmail.com 
TABLA I

CLASIFICACIÓN DE LAS GAMMAPATÍAS Y PROCESOS ASOCIADOS

\begin{tabular}{|c|c|c|c|}
\hline Tipo de gammapatía & Procesos malignos asociados & Procesos benignos asociados & $\begin{array}{l}\text { Procesos de significado incierto } \\
\text { asociados }\end{array}$ \\
\hline Gammapatía monoclonal & $\begin{array}{l}\text {-MM (IgG, IgA, IgD, IgE y } \\
\text { cadenas ligeras kappa o lambda) } \\
\text {-Plasmocitoma } \\
\text {-MW } \\
\text {-SLP (linfoma, LLC) } \\
\text {-Enf. de cadenas pesadas } \\
\text { (gamma, alfa, mu) } \\
\text {-AL } \\
\text {-Enf. por depósito de } \\
\text { inmunoglobulinas } \\
\text {-Ca (mama, colon, próstata) }\end{array}$ & $\begin{array}{l}\text {-Enf. autoinmunes (crioglobulinemia, } \\
\text { aglutininas frías, Sd. Sjögren) } \\
\text {-Enf. endocrinológicas y similares } \\
\text { (HPT, timoma, HLP, hipofosfatemia, } \\
\text { tiroiditis de Hashimoto) } \\
\text {-Enf. infecciosas (sarcoidosis, } \\
\text { parasitosis, artritis séptica) } \\
\text {-Enf. hepáticas (Cirrosis biliar primaria, } \\
\text { hepatitis crónica activa, hepatitis C) } \\
\text {-Angioedema y déficit adquirido } \\
\text { del inhibidor de C1 } \\
\text {-Enf. hematológicas (púrpura fulminante, } \\
\text { púrpura de Schönlein-Henoch, } \\
\text { esferocitosis hereditaria, Sd. de fragilidad } \\
\text { capilar sistémica) } \\
\text {-Implantes de silicona en mujeres. } \\
\text {-Enf. pulmonares (FPI, PA, HPI) }\end{array}$ & $\begin{array}{l}\text {-GMSI (IgG, IgA, IgM, IgD, cadenas } \\
\text { ligeras libres) } \\
\text {-Biclonales } \\
\text {-Proteinuria de Bence-Jones idiopática } \\
\text {-Asociadas a neoplasias de células } \\
\text { que no sean productoras de } \\
\text { proteínas monoclonales }\end{array}$ \\
\hline Gammapatía policlonal & -Enf. hematológicas (5\%) & $\begin{array}{l}\text {-Hepatopatías }(\approx 60 \%) \\
\text { - Conectivopatías }(\approx 20 \%) \\
\text { Infecciones crónicas } \approx 5 \%) \\
\text { Enfermedades hematológicas (3\%) }\end{array}$ & \\
\hline
\end{tabular}

MM: mieloma múltiple; MW: macroglobulinemia de Waldeström; AL: amiloidosis primaria; Enf: enfermedad; Ca: carcinoma; SLP: síndromes linfoproliferativos; HPT: hiperparatiroidismo; HLP: hiperlipoproteinemia; FPI: fibrosis pulmonar idiopática; PA: proteinosis alveolar; HPI: hemosiderosis pulmonar idiopática; GMSI: gammapatía monoclonal de significado incierto.

coincidir con las áreas de la beta o gama globulinas; cuando el pico monoclonal es del tipo IgF o IgE; en la enfermedad de cadenas pesadas; y en la proteinuria de Bence Jones. Una vez identificado el tipo de proteína monoclonal, no es necesario repetir la inmunoelectroforesis en sucesivas ocasiones, a no ser que se sospeche la aparición de un nuevo tipo de pico monoclonal (1).

Ambas técnicas se emplean tanto en sangre como en orina, aunque hay unas indicaciones específicas del estudio electroforético e inmunoforético en orina: si el pico monoclonal en sangre es mayor a $1,5 \mathrm{~g} / \mathrm{dl}$, si el diagnóstico es de amiloidosis primaria, si existe macroglobulinemia de Waldeström, o si el paciente ha sido diagnosticado de mieloma múltiple, para completar el estudio de dicha patología.

\section{MANEJO DEL PACIENTE CON PICO MONOCLONAL}

Además de las discordancias en las técnicas de laboratorio, el MM se diferencia de la GMSI por la presencia de una serie de síntomas como son la anemia, la hipercalcemia, la insuficiencia renal y las lesiones líticas. Sin embargo, la presencia de un índice de células plasmáticas elevado (cell labeling index) en un paciente con MM asintomático (Smoldering Multiple Myeloma o SMM) o con GMSI requiere de un seguimiento más estricto y más frecuente, por la probabilidad de progresión. Ningún otro factor puede diferenciar a un paciente con GMSI de otro en el que se desarrolle una enfermedad maligna de células plasmáticas. Los niveles séricos de proteína mono- clonal deben medirse periódicamente y debe realizarse una evaluación clínica para determinar si se ha desarrollado una enfermedad grave. Si el pico monoclonal sérico es inferior a $1,5 \mathrm{~g} / \mathrm{dl}$, debe repetirse la electroforesis en unos 6-12 meses y, si dicho pico permanece estable, se repetirá anualmente. En esta situación, no es necesario realizar radiografías óseas, estudio de médula ósea ni inmunofijación de orina de 24 horas. Si este mismo paciente, asintomático, muestra un pico monoclonal de 1,5-2,0 g/dl, deben medirse los niveles de $\operatorname{IgG}, \operatorname{IgA}$ e IgM, y se debe obtener una muestra de orina de 24 horas para hacer electroforesis y electrofijación (inmunofijación). La electroforesis se debe repetir a los 3-6 meses, y si los resultados permanecen estables, las pruebas se repetirán a los 6-12 meses, y luego, anualmente o antes si aparecen síntomas. Si los valores de IgG o IgA son superiores a 2,0 g/dl, debe realizarse una serie ósea que incluya huesos largos (húmero y fémur bilateral). También debiera llevarse a cabo una aspiración de médula ósea y una biopsia, y si fuera posible, estudios citogenéticos y búsqueda de células plasmáticas circulantes en sangre periférica. Si el pico monoclonal es muy superior a 2,0 $\mathrm{g} / \mathrm{dl}$, debe realizarse aspiración y biopsia de la médula ósea y un TAC toraco-abdominal con el fin de identificar una macroglobulinemia o procesos linfoproliferativos asociados. También se debe determinar los niveles de beta2-microglobulina y de proteína $\mathrm{C}$ reactiva. $\mathrm{Si}$ los resultados de estos tests son satisfactorios, se repetirá la electroforesis en sangre a los 3-6 meses. Si los niveles de pico monoclonal permanecen estables, el test se repetirá en 6-12 meses y después anualmente, a no ser que aparezcan síntomas o signos de macroglobulinemia (2). 


\section{GAMMAPATÍAS MONOCLONALES BENIGNAS}

Son múltiples los estudios que describen la existencia de procesos benignos que se asocian a un pico monoclonal en sangre. Arnulf B y cols. (3) realizaron un estudio prospectivo que indica que la prevalencia de GMSI es mayor en los pacientes con hiperparatiroidismo, por lo que debería realizarse una electroforesis sérica en todos los pacientes con dicha endocrinopatía; a su vez, en los pacientes con GMSI en los que se detecte hipercalcemia sin otros síntomas acompañantes, debiera hacerse un despistaje de hiperparatiroidismo.

También diversas enfermedades hematológicas benignas pueden asociarse a una gammapatía monoclonal. Un ejemplo lo constituye la enfermedad de von Willebrand adquirida. Su asociación con un pico monoclonal en sangre es tan frecuente, que debiera hacerse el despistaje de gammapatía monoclonal en todo adulto con este diagnóstico (4). Otras enfermedades hematológicas asociadas son el síndrome antifosfolípido, la anemia perniciosa, la mielofibrosis o la enfermedad de Gaucher.

En algunas conectivopatías, como la artritis reumatoide, la espondilitis anquilosante, la polimialgia reumática, el lupus eritematoso, la esclerodermia o la polimiositis, llega a detectarse un pico monoclonal.

Las enfermedades dermatológicas son otro grupo de procesos que pueden acompañarse de la presencia de un pico monoclonal. Así, la mucinosis papular o el xantogranuloma necrobiótico, se relacionan con las cadenas lambda IgG; el síndrome de Schnitzler, al pico monoclonal IgM, o el pioderma gangrenoso o el escleredema de Buschke, a otros componentes monoclonales. Algunos procesos malignos, como la micosis fungoide o el sarcoma de Kaposi, pueden cursar como gammapatías monoclonales.

En los pacientes con el síndrome de inmunodeficiencia adquirida es bastante frecuente que exista gammapatía monoclonal, que no suele tener importancia pronóstica. Lu C y cols. aconsejan el despistaje de infección por VIH en todos los pacientes con GMSI que hayan podido estar expuestos al virus (5).

Existe multitud de enfermedades y patologías benignas en las que se puede asociar un pico monoclonal en sangre. La descripción de algunas de ellas aparece en la tabla I.

\section{GAMMAPATÍA MONOCLONAL DE SIGNIFICADO INCIERTO}

En 1952, Waldeström introdujo el término "hiperglobulinemia esencial" para describir a aquellos pacientes con un pico de inmunoglobulinas (Ig) de escasa cuantía en la electroforesis, sin que se tratara de un mieloma múltiple (MM), una macroglobulinemia de Waldeström (MW), una amiloidosis (AL) $\mathrm{u}$ otros procesos malignos relacionados. Desde entonces, se han empleado múltiples sinónimos para aquél término, destacando el de "gammapatía monoclonal de significado incierto" (GMSI). El término "gammapatía monoclonal benigna" es erróneo, porque en el momento del diagnóstico no se sabe si el proceso malignizará o no.

La definición más reciente de GMSI ha sido elaborada por The International Myeloma Working Group en 2003, e incluye una serie de criterios, que están reflejados en la tabla II (6). Aunque son numerosas las series que informan acerca de la prevalencia de este proceso, recientemente, Kyle y cols. han
TABLA II

CRITERIOS DIAGNÓSTICOS DE LAS PRINCIPALES GAMMAPATÍAS MONOCLONALES SEGÚN THE INTERNACIONAL MYELOMA WORKING GROUP (6)

- Criterios diagnósticos de la GMSI

- Pico monoclonal $<3 \mathrm{~g} / \mathrm{dl}$

- $<10 \%$ células plasmáticas en biopsia de médula ósea

- No evidencia de otros procesos proliferativos de células B

- No proteína monoclonal en orina, o concentración < 50 mg/día

- No anemia asociada

- No hipercalcemia asociada

- No insuficiencia renal asociada

-Criterios diagnósticos del SMM

- Pico monoclonal en sangre $\geq 30 \mathrm{~g} / \mathrm{l} / \mathrm{o}$

- $\geq 10 \%$ de células plasmáticas en médula ósea

- No afectación de órganos ni de tejidos, ni síntomas asociados a estos

-Criterios diagnósticos del MM sintomático

- Pico monoclonal en sangre y/u orina

- Infiltración de la médula ósea por células plasmáticas o plasmocitoma

- Afectación de órganos o tejidos relacionados (incluyendo lesiones óseas): anemia, hipercalcemia, lesiones óseas líticas, insuficiencia renal, hiperviscosidad, amiloidosis o infecciones recurrentes

\section{- Criterios diagnósticos del plasmocitoma solitario}

- Ausencia de pico monoclonal en sangre y orina (a veces existe un pico monoclonal, pero de escasa cantidad)

- Una sola área de destrucción ósea

- Médula ósea no compatible con la del MM

- Estudios radiológicos normales

- No daño de los órganos principales (salvo la lesión ósea solitaria)

-Criterios diagnósticos del plasmocitoma solitario recurrente

(múltiple)

- Ausencia de pico monoclonal en sangre y orina (a veces existe un pico monoclonal, pero de escasa cantidad)

- Más de un área de destrucción ósea o tumor extramedular de células plasmáticas monoclonales que pueda recurrir

- Médula ósea normal

- Estudios radiológicos normales

- No daño de los órganos principales

- Criterios diagnósticos del mieloma no secretor

- Ausencia de pico monoclonal en sangre y orina

- Lesiones óseas

- Médula ósea con $\geq 10 \%$ de células plasmáticas

- Afectación de órganos principales

GMSI: gammapatía monoclonal de significado incierto; SMM: Smoldering multiple myeloma; MM: mieloma múltiple.

reportado los datos relativos a la población de Minnesota, que englobaba a 21.463 personas: la prevalencia es del 3,2\% en pacientes con 50 años o más y 5,3\% de las personas con 70 años o más (7).

La GMSI no produce síntomas y suele detectarse durante exámenes rutinarios en pacientes aparentemente normales, o durante la evaluación de otros procesos no relacionados. Es 
importante para los médicos y para el paciente determinar si la gammapatía monoclonal permanece estable (es benigna) o si progresa a MM o procesos relacionados (existe riesgo de transformación a un mieloma múltiple incluso 30 años tras el diagnóstico de GMSI). Como no es posible predecir cuándo se producirá la transformación maligna, o incluso si llegará a producirse o no, los pacientes que tengan una gammapatía monoclonal de significado incierto deben ser seguidos durante un largo periodo de tiempo.

\section{FACTORES PREDICTIVOS DE MALIGNIZACIÓN DE LA GMSI}

Ninguno de los hallazgos encontrados en el momento del diagnóstico de la GMSI permite distinguir entre los pacientes con posibilidad de permanecer estables y los pacientes en los que se producirá una evolución a malignización. Cuando una GMSI maligniza a MM o a MW, el tipo de proteína monoclonal sigue siendo la misma. Hasta ahora, los factores predictivos identificados son:

1. El tamaño de la proteína monoclonal:

En una serie de Kyle y Rajkumar de la Clínica Mayo (2) que englobaba a 1384 pacientes, la cantidad de proteína monoclonal detectada en el momento del diagnóstico de la GMSI era el factor predictor más importante de progresión a enfermedad de células plasmáticas. Así, si el pico es $\leq 0,5$ $\mathrm{g} / \mathrm{dl}$, el porcentaje de progresión a mieloma múltiple a los 10 años se aproxima al 5\%, y tras 20 años de seguimiento, al $15 \%$; por el contrario, si el pico monoclonal es de 3,0 g/dl, la progresión a mieloma múltiple a los 10 años será próxima al $35 \%$, y a los 20 años, al $65 \%$.

2. El tipo de inmunoglobulina:

En la serie del sudeste de Minnesota (8), los pacientes con pico monoclonal IgM o IgA tenían un mayor riesgo de progresión en comparación con aquellos con proteína $\operatorname{IgG}(\mathrm{p}=0,001)$.

3. La proporción de células plasmáticas de la médula ósea:

En un estudio realizado por Baldini y cols. (9), el porcentaje de progresión fue del 6,8\% en los pacientes cuyo nivel de células plasmáticas en médula ósea era inferior al $10 \%$ y del $37 \%$ si tenían un 10-30\% de células plasmáticas en médula ósea. Cesana y cols. (10) hicieron otro estudio con el que concluían que la presencia de más de un $5 \%$ de células plasmáticas en la médula ósea era un factor de riesgo independiente para la progresión.

4. Otros:

Según Kyle y Rajkumar (2), en los 1.384 pacientes que estudiaron en la Clínica Mayo, reportaron que la presencia de proteinuria de Bence Jones y la elevación de la VSG eran factores independientes de progresión en la GMSI.

La GMSI en la que predomina la IgM suele progresar a linfoma o a MW, mientras que la tipo IgA o IgG suelen progresar a MM o, a $\mathrm{AL}$ o a otros procesos relacionados con las células plasmáticas (11).

\section{CRIOGLOBULINEMIA}

La crioglobulinemia es una forma especial de vasculitis sistémica, englobada en el subgrupo de las vasculitis mediadas por complejos inmunes.

La clasificación de las crioglobulinemias fue llevada a cabo por Brouet en 1983 (Tabla III).
TABLA III

CLASIFICACIÓN DE LAS CRIOGLOBULINEMIAS Y PROCESOS ASOCIADOS

\begin{tabular}{lll}
\hline $\begin{array}{l}\text { Tipo de } \\
\text { crioglobulinemia }\end{array}$ & Características & Procesos asociados \\
\hline $\begin{array}{l}\text { Tipo I (crioglobulinemia } \\
\text { monoclonal) }\end{array}$ & $\begin{array}{l}\text { Ig monoclonales (IgM } \\
\text { la más frecuente) }\end{array}$ & $\begin{array}{l}\text { Sd. linfoproliferativos } \\
\text { (MW, MM, LLC, } \\
\text { linfomas) }\end{array}$ \\
$\begin{array}{l}\text { Tipo II (crioglobulinemia } \\
\text { mixta) }\end{array}$ & $\begin{array}{l}\text { Ig monoclonales } \\
\text { anti-|gC y policlonales } \\
(I g G)\end{array}$ & $\begin{array}{l}\text { Enfermedades auto- } \\
\text { inmunes, Sd. linfopro- } \\
\text { liferativos, infecciones } \\
\text { crónicas, enfermedades } \\
\text { del tejido conectivo }\end{array}$ \\
$\begin{array}{l}\text { Tipo III (crioglobulinemia } \\
\text { mixta) }\end{array}$ & Ig policlonales & Los mismos que el tipo II \\
\hline
\end{tabular}

Sd: síndrome; MM: mieloma múltiple; MW: macroglobulinemia de Waldeström; LLC: leucemia linfática crónica.

En la mayoría de los casos es de etiología desconocida (enfermedad de las crioaglutininas idiopática), y es especialmente típica de personas de edad avanzada.

La crioglobulinemia mixta tipo II está ampliamente causada por el virus de la hepatitis C (VHC), pero éste, además, es la causa principal de los 3 tipos de crioglobulinemias y el tipo de Ig asociada.

Las manifestaciones clínicas más frecuentes de la crioglobulinemia monoclonal son la púrpura no palpable en extremidades inferiores, secundaria a la formación de trombos en el interior de los vasos o infartos hemorrágicos, con úlceras también secundarias a la trombosis intravascular, en dedos de las manos y de los pies. Estas manifestaciones se exacerban tras la exposición al frío o el aumento de la viscosidad sanguínea.

Las manifestaciones clínicas de la crioglobulinemia mixta oscilan desde una vasculitis asintomática o casi asintomática, con púrpura palpable, artralgias y fatiga, hasta una vasculitis severa, con necrosis cutánea, afectación renal, afectación de los nervios periféricos, del sistema nervioso central, del aparato respiratorio, del miocardio y/o del tracto gastrointestinal.

\section{GAMMAPATÍAS MONOCLONALES MALIGNAS O DE COMPORTAMIENTO AGRESIVO}

\section{SÍNDROME POEMS}

Este síndrome fue descrito por Bardwick y cols. (12) en 1980, y consiste en la asociación de Polineuropatía, Organomegalia, Endocrinopatía, pico Monoclonal ( $\operatorname{IgA}$ o $\operatorname{IgG}$ tipo lambda en el $75 \%$ de los casos) y cambios en la piel (Skin). Recientemente, Dispenzieri, et al han intentado definir los criterios diagnósticos del síndrome POEMS, que son los siguientes (13):

-Criterios mayores:

- Polineuropatía, ascendente y simétrica, que suele ser el síntoma de presentación.

- Alteración monoclonal por proliferación de células plasmáticas, que no suelen superar el $5 \%$ en el aspirado de la médula ósea. 
- Criterios menores:

- Lesiones óseas escleróticas, que pueden ser solitarias o múltiples.

- Enfermedad de Castleman, aunque no se conoce bien por qué se produce esta asociación.

- Organomegalia (esplenomegalia, hepatomegalia o linfadenopatía).

- Edema (edema, derrame pleural o ascitis).

- Endocrinopatía (suprarrenal, tiroidea, hipofisaria, gonadal, paratiroidea, pancreática). Debido a la alta prevalencia de diabetes mellitus y de anomalías tiroideas, este diagnóstico solo no es suficiente para cumplir el criterio menor de endocrinopatía.

- Cambios cutáneos (hiperpigmentación, hipertricosis, plétora facial, hemangiomas).

- Papiledema, que puede acompañarse de cefalea.

Se requiere al menos 1 criterio mayor y 2 criterios menores para llegar al diagnóstico.

El pronóstico de estos pacientes, según la mayoría de los estudios, es muy pobre, con una supervivencia media estimada de 12-33 meses.

En pacientes con neuropatía periférica inexplicada, debe determinarse la electroforesis en sangre y orina, con inmunofijación incluida, el aspirado de médula ósea, y un examen cuidadoso de piel, visceromegalias, ganglios linfáticos y papila del nervio óptico, así como un estudio de lesiones óseas. Si la sospecha clínica es muy alta, debiera llevarse a cabo una evaluación endocrinológica y si el paciente tiene síntomas respiratorios, se hará tests de función pulmonar y ecocardiografías (13).

\section{MIELOMA MÚLTIPLE}

Este proceso ha sido redefinido recientemente, en el año 2003, sustituyéndose la definición clásica enunciada por Durie y Salmon. En la tabla II aparecen las nuevas definiciones de las distintas variantes del mieloma múltiple (6).

\section{ENFERMEDAD DE LAS CADENAS PESADAS}

Familia de enfermedades raras, de clínica linfoma-like, en las que existe producción de componente monoclonal de Ig en ausencia de cadenas ligeras. Si el componente monoclonal es tipo IgG, se asemeja al linfoma de Hodgkin; si es tipo IgA, puede infiltrar el intestino delgado y causar malabsorción, y cuando es tipo IgM, se asemeja a una leucemia linfática crónica (1).

\section{AMILOIDOSIS}

La amiloidosis es un "trastorno" de clones de células plasmáticas o linfocitos que producen una Ig monoclonal. Esto conduce al depósito de una proteína fibrilar en múltiples órganos. En la médula ósea de estos pacientes se detecta un porcentaje bajo de células plasmáticas (como en la GMSI) y suelen estar implicadas cadenas ligeras tipo lambda del subgrupo VI. La edad media al diagnóstico de 60 años y la supervivencia media depende de los órganos afectos y de la existencia de MW (1).

\section{ENFERMEDAD POR DEPÓSITO DE IG}

Esta enfermedad es secundaria al depósito de cristales de Ig monoclonal o sus componentes en determinados tejidos. Las mayores complicaciones se deben al depósito de dichas Ig en los glomérulos, causando un síndrome nefrótico, y posteriormente, insuficiencia renal.

Hasta ahora, se ha enunciado las principales patologías que se asocian a la detección de una proteína monoclonal sérica. En la tabla IV se indica, de forma esquemática los principales parámetros de laboratorio y de técnicas de imagen que pueden ayudar a diferenciar las distintas patologías. Es crucial llegar a un diagnóstico diferencial adecuado ante la presencia de un pico monoclonal, que influirá en la futura evolución y en la supervivencia del paciente.

TABLA IV

DIAGNÓSTICO DIFERENCIAL ENTRE LA GAMMAPATÍA MONOCLONAL DE SIGNIFICADO INCIERTO, LAS DISTINTAS VARIANTES DEL MIELOMA, LA AMILOIDOSIS Y LA MACROGLOBULINEMIA DE WALDESTRÖM

\begin{tabular}{|c|c|c|c|c|c|c|c|}
\hline & Pico en sangre & Pico en orina & $B M O$ & Anemia & Hipercalcemia & Irenal & Afectación ósea \\
\hline GMSI & $<3 \mathrm{~g} / \mathrm{dl}$ & $00<50 \mathrm{mg} / \mathrm{d}$ & $<10 \%$ & No & No & No & No \\
\hline SMM & $>3 \mathrm{~g} / \mathrm{dl}$ & & $>10 \%$ & No & No & No & No \\
\hline$M M$ & $>3 \mathrm{~g} / \mathrm{dl}$ & $>50$ & $>10 \%$ & Sí & Sí & Sí & Sí \\
\hline MNS & 0 & 0 & $>10 \%$ & Sí & Sí & Sí & Sí \\
\hline PS & 0 & 0 & $<10 \%$ & 0 & 0 & 0 & Sí \\
\hline PM & 0 & 0 & $<10 \%$ & 0 & 0 & 0 & Sí \\
\hline MW & Cualquiera & Cualquiera & $\begin{array}{l}\text { Patrón } \\
\text { intertra-becular }\end{array}$ & Sí (> 50\%) & No & Rara & $10 \%$ osteoporosis \\
\hline$A M$ & Cualquiera (90\%) & Cualquiera (75\%) & $<10 \%(80 \%)$ & $+/-$ & No & Sd. nefrótico & No \\
\hline
\end{tabular}

GMSI: gammapatía monoclonal de significado incierto; SMM: Smoldering multiple myeloma; MM: mieloma múltiple; MNS: mieloma no secretor; PS: plasmocitoma solitario; PM: plasmocitoma múltiple; MW: macroglobulinemia de Waldeström; AM: amiloidosis primaria; BMO: biopsia de médula ósea; I. renal: insuficiencia renal. 


\section{Bibliografía}

1. Alexanian R, Weber D, Liu F. Differential diagnosis of monoclonal gammopathies. Arch Pathol Lab Med 1999; 123: 108-113.

2. Kyle RA, Rajkumar SV. Monoclonal gammopathies of undetermined significance: a review. Inmunol Rev 2003; 194: 112-139.

3. Arnulf B, Bengoufa D, Sarfati E, Toubert ME, Meignin V, Brouet JC, et al. Prevalence of monoclonal gammopathy in patients with primary hyperparathyroidism: a prospective study. Arch Intern Med, 2002; 162: 464-7.

4. Lamboley V, Zabraniecki L, Sie P, Pourrat J, Fournie B. Myeloma and monoclonal gammopathy of uncertain significance associated with acquired von Willebrand's syndrome. Seven new cases with a literature review. Joint Bone Spine 2002; 69: 62-7.

5. Lu CH, Dezube BJ, Pantanowitz L. HIV infection masquerading as monoclonal gammopathy of undetermined significance. N Eng J Med 2003; 349: 1192-3.

6. The International Myeloma Working Group. Criteria for the classification of monoclonal gammopathies, multiple myeloma and related disorders: a report of the International Myeloma Working Group. Br J Haematol 2003; 121: 749-757.

7. Kyle RA, Therneau TM, Rajkumar V, Larson DR, Plevak MF, Oxford JR, et al. Prevalence of monoclonal gammopathy of undetermined sig- nificance. N Engl J Med 2006; 354: 1362-9.

8. Kyle RA, Therneau TM, Rajkumar SV, Oxford JR, Larson DR, Plevak $\mathrm{MF}$, et al. A long-term study of prognosis in monoclonal gammopathy of undetermined significance. N Engl J Med 2002; 346: 564-569.

9. Baldini L, Guffanti A, Cesana BM, Colombi M, CHiorboli O, Damilano I, et al. Role of different hematologic variables in defining the risk of malignant transformation in monoclonal gammopathy. Blood 1996; 87: 912-918

10. Cesana C, Klersy C, Barbarazo L, Nosari AM, Crugnola M, Pungolino E, et al. Prognostic factors for malignant transformation in monoclonal gammopathy of undetermined significance and smoldering multiple myeloma. J Clin Oncol 2002; 20: 1625-1634.

11. Kyle RA, Therneau TM, Rajkumar SV, Remstein ED, Offord JR, Larson DR, et al. Long-term-follow-up of IgM monoclonal gammopathy of undetermined significance. Blood 2003; 102: 3759-3764.

12. Bardwick PA, Zvaiffer NJ, Gill GN, Newman D, Greenway GD, Resnick DL. Plasma cell dyscrasia with polineruropathy, organomegaly, endocrinopathy, $\mathrm{M}$ protein and skin changes: the POEMS syndrome. Report on two cases and a review of the literature. Medicine (Baltimore) 1980; 59: 311-322.

13. Dispenzieri A. POEMS Syndrome. Hematology 2005; 360-7. 